\title{
The Influence of Amyloid Burden on Cognitive Decline over 2 years in Older Adults with Subjective Cognitive Decline: A Prospective Cohort Study
}

\author{
Yun Jeong Hong ${ }^{\mathrm{a}}$ Jeong Wook Park ${ }^{\mathrm{a}}$ Si Baek Lee ${ }^{\mathrm{a}}$ Seong-Hoon Kim ${ }^{\mathrm{a}}$ \\ Yongbang Kim $^{\mathrm{a}}$ Dong-Woo Ryu ${ }^{\mathrm{b}}$ Kyung Won Park ${ }^{\mathrm{c}}$ Dong Won Yang ${ }^{\mathrm{d}}$
}

${ }^{a}$ Neurology, Uijeongbu St. Mary's Hospital, The Catholic University of Korea, Seoul, Republic of Korea; ${ }^{b}$ Neurology, The Catholic University of Korea, Seoul, Republic of Korea; 'Neurology, Dong-A University College of Medicine, Busan, Republic of Korea; ${ }^{d}$ Neurology, Seoul St. Mary's Hospital, The Catholic University of Korea, Seoul, Republic of Korea

\section{Keywords}

Subjective cognitive decline - Alzheimer's disease .

Neuroimaging biomarker $\cdot$ Cognitive decline $\cdot$ Amyloidosis . Amyloid positron emission tomography

\begin{abstract}
Background: Subjective cognitive decline (SCD) is a self-perceived cognitive worsening without objective cognitive impairment. Due to its heterogeneity and potential risk of Alzheimer's disease (AD), baseline biomarkers to predict progression are clinically important. In the present study, cognitive trajectories during a 24-month period were compared between amyloid-positive SCD $(A+S C D)$ and amyloidnegative SCD (A-SCD) subjects, and biomarkers associated with memory decline were investigated. Methods: Data from a prospective cohort study in Korea between 2016 and 2019 were analyzed. SCD subjects $\geq 50$ years of age were eligible. All participants underwent neuropsychological tests, brain magnetic resonance imaging, and florbetaben positron emission tomography scans. Amyloid burden and regional volumes were measured. Cognitive changes corrected for age were compared between $\mathrm{A}+\mathrm{SCD}$ and $\mathrm{A}-\mathrm{SCD}$ groups. Biomarkers associated with memory decline were assessed. $\boldsymbol{R e}$ sults: Forty-seven SCD subjects (69.9 \pm 6.7 years, mini-mental
\end{abstract}

state examination (MMSE) score 27.5) were enrolled, and 31 completed at least 1 annual follow-up (mean follow-up: 24.7 months). Baseline characteristics except age, hippocampal atrophy, and white matter hyperintensities were similar between A+SCDs $(n=12,25.6 \%)$ and A-SCDs $(n=35)$. A+SCD subjects showed greater decline in the verbal memory function compared with the A-SCD subjects after adjustment for age. MMSE scores decreased more in the A+SCD (1.1 in the $\mathrm{A}+\mathrm{SCD} ; 0.55$ in the $\mathrm{A}-\mathrm{SCD}$ ), although it was not statistically significant. Amyloid burden and baseline memory score were associated with memory decline. Conclusions: Within $S C D$, $A+S C D$ subjects showed faster memory decline compared with the A-SCD subjects and amyloid burden might be associated with future memory decline in SCD.

(c) 2021 The Author(s).

Published by S. Karger AG, Basel

\section{Introduction}

Subjective cognitive decline (SCD) is characterized by self-perceived cognitive worsening in the absence of objective cognitive impairment. Cognitively normal individuals with SCD are thought to have higher risk of Alzheimer's disease (AD) dementia [1-5]. Because selfawareness and concerns regarding minor cognitive karger@karger.com www.karger.com/dem

Karger $\stackrel{\text { ' }}{5}$
(C) 2021 The Author(s)

Published by S. Karger AG, Basel

This is an Open Access article licensed under the Creative Commons Attribution-NonCommercial-4.0 International License (CC BY-NC) (http://www.karger.com/Services/OpenAccessLicense), applicable to the online version of the article only. Usage and distribution for commercial purposes requires written permission.
Correspondence to:

Yun Jeong Hong, hyj2009@ hanmail.net 
decline could be an early sign of $\mathrm{AD}$, subjects with $\mathrm{SCD}$ are the most appropriate target population for secondary preventions. However, due to the heterogeneity of both pathologies and cognitive trajectories, baseline clinical and biomarker findings to predict future cognitive decline are clinically important in this stage.

In recent studies, old age, apolipoprotein $\varepsilon 4$ (APOE4) existence, and amyloid pathologies at baseline were reportedly relevant factors for cognitive decline in subjects with SCD [3, 6-8]. In SCD with amyloidosis, the risk of clinical progressions increases to $40-62 \%$ during approximately 3 years [5], which might be why SCD with amyloidosis is considered as the later stages of preclinical AD. In our previous cross-sectional data [9], amyloid-positive SCD $(\mathrm{A}+\mathrm{SCD})$ subjects differed from amyloid-negative SCD (A-SCD) in baseline small vessel disease markers, degree of hippocampal atrophy, and a few clinical features (existence of SCD-plus clinical features) regarding cognitive complaints $[9,10]$. The results showed that A+SCD participants are different in baseline status and characteristics in regard to the complaints; however, it was not assessed whether the baseline amyloidosis is associated with future cognitive decline.

In this prospective observational cohort study, we included individuals diagnosed as SCD from a memory clinic cohort and underwent amyloid positron emission tomography (PET) scans and brain magnetic resonance imaging (MRI) to assess amyloid burden and neurodegenerations, with regular follow-up evaluations including MRI and cognitive tests during a 24-month period. In the present study, cognitive trajectories between $\mathrm{A}+\mathrm{SCD}$ and A-SCD subjects were compared and relationships between baseline biomarkers and memory decline were measured to investigate whether amyloid burden is associated with future cognitive decline.

\section{Materials and Methods}

\section{Participants}

This study was performed at a university-affiliated dementia clinic from December 2016 to July 2019. Elderly subjects who visited the hospital due to cognitive worsening and were diagnosed with SCD after dementia work-up were consecutively recruited during the study period. The dementia work-up included detailed neuropsychological test battery, MRI, and routine blood sampling for syphilis, thyroid function, vitamin B deficiencies, and APOE genotyping. The study inclusion criteria were the following: (1) existence of persistent self-reported cognitive complaints; (2) older than 50 years of age; (3) normal performance (above -1.0 standard deviation of norms) on all subtests of neuropsychological test battery named Seoul Neuropsychological Screening Battery (SNSB) [11]; (4) clinical dementia rating (CDR) [12] score of 0; (5) literate; and (6) agreement to partici- pate in the study and could visit the hospital for annual evaluations. The exclusion criteria were the following: (1) any unstable or severe medical condition (e.g., severe hepatic or renal disease, unstable cardiovascular disease, severe asthma, active gastric ulcer, cancer); (2) neurological disorders such as Parkinson's disease, Huntington's disease, or normal pressure hydrocephalus; (3) major psychiatric disorders such as uncontrolled depression, schizophrenia, alcoholism, or drug dependency; (4) mild cognitive impairment (MCI) or dementia; (5) abnormal blood laboratory findings such as abnormal thyroid function, low vitamin B12 or low folate, or positive syphilis serology; and (6) brain lesions known to cause cognitive impairment (tumor, stroke, or subdural hematoma). All study participants underwent baseline physical and neurological examinations and florbetaben PET scans for amyloidosis. No pharmacological treatments or nonpharmacological interventions were administered to the participants. All participants underwent annual follow-up evaluations to assess cognitive decline/clinical progression, and brain MRI scans were performed at the second follow-up visit (after 24 months from the baseline) to assess atrophic changes during the study period. This study was approved by the Institutional Review Board (DAUHIRB-16-232), and written informed consent was obtained from all participants. The study was conducted in accordance with the Declaration of Helsinki and principles of Good Clinical Practice.

\section{Neuroimaging}

Brain MRI was performed using a 3.0-Tesla scanner (GE Medical Systems, Milwaukee, WI, USA), including fluid attenuated inversion recovery (FLAIR), gradient echo, and 3-dimensional (3D) T1-weighted images. The white matter hyperintensities (WMHs) were rated using a visual rating scale of axial FLAIR images. In brief, periventricular WMHs and deep WMHs were evaluated separately and rated as minimal (grade 1), moderate (grade 2), or severe (grade 3) [13]. Lacunes were defined as small lesions (3-15 $\mathrm{mm}$ in diameter), hyperintense on T2-, and hypointense on T1weighted images, with a perilesional halo on FLAIR [14]. Cerebral cortical microbleeds were defined as round and homogeneously low-signal lesions $<10 \mathrm{~mm}$ in diameter in lobar areas on gradient echo images [14]. Hippocampal atrophy was rated on coronal T1weighted images using Scheltens' visual rating scale [15]. The mean of the left and right hippocampal atrophy scores was used. The number of lacunes, number of microbleeds, degree of $\mathrm{WMH}$, and degree of hippocampal atrophy were measured by a neurologist (YB Kim) blinded to the data.

Florbetaben (18F) PET scans were acquired following the standardized protocol [16]. Using PET scans, a whole brain visual interpretation was performed by a trained specialist in nuclear medicine who was blinded $t$ the patient diagnosis. A visual rating scale of florbetaben PET scans, the brain amyloid plaque load score (1 = negative finding, $2=$ moderate amyloid depositions, $3=$ pronounced amyloid depositions) [17], was used to categorize amyloid-positive and amyloid-negative participants. Brain amyloid plaque load scores of 2 or 3 indicated a positive finding for amyloidosis $(\mathrm{A} \beta+)$.

In addition, quantitative neuroimaging analyses were performed using PET scans and MRI 3D T1 images. First, amyloid depositions were assessed using MATLAB version 2013a and SPM8 (http://www.fil.ion.ucl.ac.uk/spm/software/spm8). Individual 3D T1-weighted MRI scans were estimated and co-registered into corresponding PET images. A volume-based template, incorporating 90 regions of interest (ROI), named automatic anatomical labeling 
Fig. 1. Flowchart of the participants. SCD, subjective cognitive decline; PET, positron emission tomography.

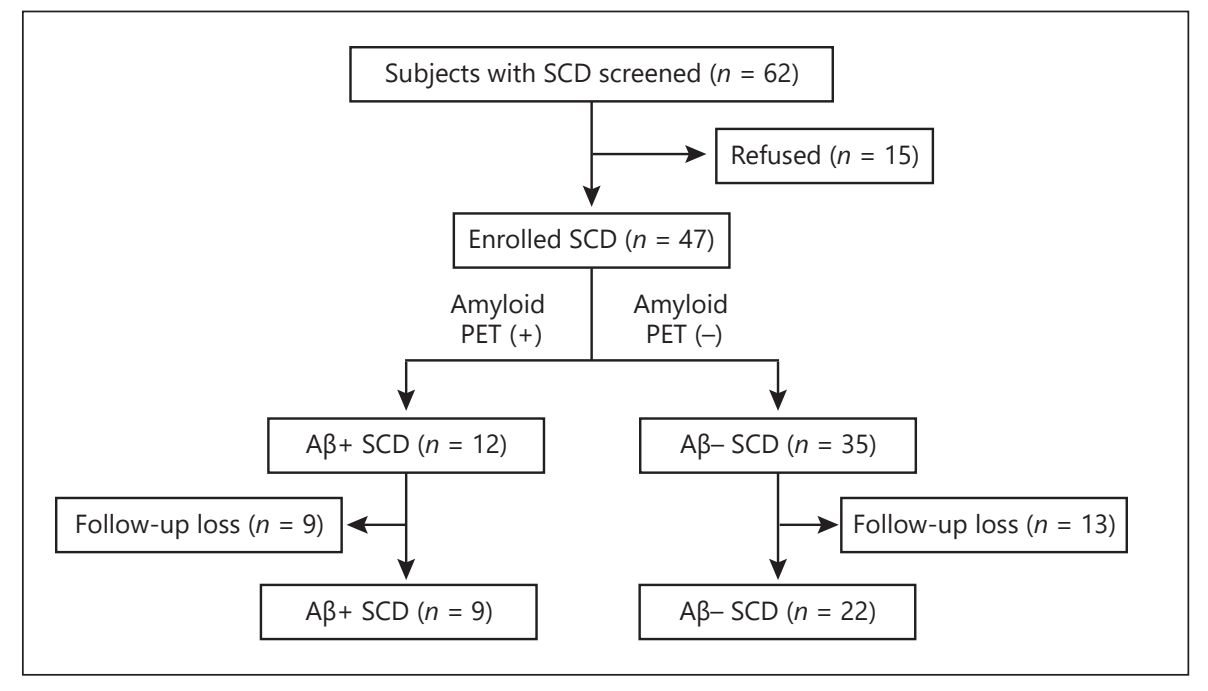

(AAL), was aligned to individual T1-weighted MRI images. The voxels of florbetaben PET images were scaled using the mean uptake value in the cerebellar cortex to calculate the standardized uptake value ratio (SUVR), and partial volume corrections were performed. For partial volume corrections, the voxels located in gray matter with a probability $<20$ percent were discarded in each PET images. We selected $28 \mathrm{AD}$-specific cortical ROIs from the AAL atlas according to the previous methods [18] and the mean SUVR values were calculated as a global SUVR. Second, the volumes of regional cortical areas including anterior frontal, dorsolateral frontal, orbitofrontal, lateral temporal, medial temporal, lateral parietal, medial parietal, and occipital lobes were measured from individual 3D T1 images using MATLAB 2013a, SPM8, and the newly developed in-house program named Quick Brain Volumetry (QBraVo). The methods of the automated volumetry program, QBraVo, are described in the online supplementary data (for all online suppl. material, see www.karger.com/doi/10.1159/000519766). To assess early neurodegenerative changes in $\mathrm{AD}$, hippocampal and entorhinal volumes were separately measured using the QBraVo.

Baseline and Follow-Up Neuropsychological Tests

All participants were diagnosed with SCD using the formal neuropsychological test battery SNSB [11], including the Korean version of the mini-mental state examination (K-MMSE) [19], $\mathrm{CDR}$, activities of daily living (ADL), attention (digit span test), language (Boston naming test, tests for comprehension/repetition/fluency), visuospatial function (Rey Complex Figure Test, RCFT), verbal and visual memory function [Seoul Verbal Learning Test (SVLT) and RCFT recall test], and frontal executive function (contrasting program, go-no-go, Controlled Oral Word Association Test, and Stroop test) [11]. Age-, sex-, and education-specific norms based on normal controls were used to interpret the SNSB results. Scores $\geq 16$ th percentile, which were compared to -1 standard deviation (SD) of the norm, were defined as normal. The severity of the cognitive complaints was assessed using a self-rated scale named Cognitive Failures Questionnaire (CFQ) (total score 0-100, higher total score means more cognitive complaints) [20]. Using a self-report questionnaire, "informant also report a cognitive decline of the participant," "subjective concern about the cog-

Faster Memory Decline in Amyloid-

Positive Subjective Cognitive Decline nitive decline," and "symptom's onset after 65 years of age" were assessed at baseline and annual follow-up evaluations because the questions are parts of "SCD-plus criteria" and were associated with Alzheimer's pathologic changes in our previous study [9].

Annual follow-up evaluations (visit window up to 3 months was allowed) included cognitive tests (K-MMSE, CDR, and verbal learning immediate/delayed/recognition tests), Korean version of the instrumental ADL (K-IADL) scale [21], neurological and physical examinations, and physician's history taking to assess clinical progression to MCI or dementia. The cognitive tests were administered by a trained neuropsychologist. Participants with CDR score $\geq 0.5$ or K-IADL score $\geq 0.43$ were considered to have progressed to MCI or dementia.

\section{Statistical Analysis}

Independent $t$-test or nonparametric Mann-Whitney $U$ test (based on normal distribution patterns) was used for comparison of continuous variables such as baseline demographics and clinical characteristics between $\mathrm{A}+\mathrm{SCD}$ and $\mathrm{A}-\mathrm{SCD}$ subjects. $\chi^{2}$ tests were used to compare categorical variables between the 2 groups. Analysis of covariance (ANCOVA) corrected for age and baseline scores was used to compare cognitive changes between the 2 groups. To assess relevant baseline factors associated with verbal memory decline, multivariable linear regression analysis was performed. All statistical analyses were performed using SPSS (version 18.0; SPSS Inc, Chicago, IL, USA). $p$ values $<0.05$ were considered to indicate statistically significant differences.

\section{Results}

\section{Baseline Demographics}

Initially, 62 subjects who were diagnosed as SCD were screened; 15 refused to participate in the study. A total of 47 participants with SCD (mean age: 69.9 \pm 6.7 years) was enrolled at baseline, and 31 subjects with SCD completed at least 1 annual follow-up evaluation (Fig. 1). Sixteen 
Table 1. Baseline characteristics and clinical findings between groups (total enrolled subjects)

\begin{tabular}{|c|c|c|c|}
\hline Variables & $\begin{array}{l}\text { Amyloid PET+ } \\
(n=12)\end{array}$ & $\begin{array}{l}\text { Amyloid PET- } \\
(n=35)\end{array}$ & $p$ value \\
\hline \multicolumn{4}{|l|}{ Demographics } \\
\hline Age, years & $74.17 \pm 4.47$ & $67.09 \pm 6.71$ & 0.001 \\
\hline Female, $n(\%)$ & $7 / 12(58.3)$ & 23/35 (65.7) & 0.733 \\
\hline Education, years & $7.83 \pm 2.86$ & $9.61 \pm 4.76$ & 0.132 \\
\hline APOE4 allele, $n(\%)$ & $5 / 12(41.7)$ & $5 / 35(14.3)$ & 0.096 \\
\hline Hypertension, $n$ (\%) & $7 / 12(58.3)$ & $14 / 35(40)$ & 0.270 \\
\hline $\mathrm{DM}, n(\%)$ & 4/12 (33.3) & $7 / 35(20)$ & 0.435 \\
\hline Hyperlipidemia, $n$ (\%) & $3 / 12(25)$ & $11 / 35(31.4)$ & 0.734 \\
\hline \multicolumn{4}{|l|}{ Neuroimaging findings } \\
\hline Global SUVR, ratio & $1.53 \pm 0.25$ & $1.17 \pm 0.06$ & 0.001 \\
\hline Hippocampal atrophy, grade & $1.17 \pm 0.94$ & $0.36 \pm 0.59$ & 0.015 \\
\hline Ant frontal vol., $\mathrm{cm}^{3}$ & $50.66 \pm 7.10$ & $56.39 \pm 8.31$ & $0.045^{*}$ \\
\hline Dorsolateral frontal vol., $\mathrm{cm}^{3}$ & $67.34 \pm 8.86$ & $71.75 \pm 10.76$ & 0.225 \\
\hline Orbitofrontal vol., $\mathrm{cm}^{3}$ & $33.47 \pm 4.64$ & $37.11 \pm 5.89$ & 0.068 \\
\hline Ant temporal vol., $\mathrm{cm}^{3}$ & $30.45 \pm 4.38$ & $32.01 \pm 5.42$ & 0.392 \\
\hline Med temporal vol., $\mathrm{cm}^{3}$ & $31.14 \pm 3.00$ & $32.47 \pm 3.58$ & 0.272 \\
\hline Lat temporal vol., $\mathrm{cm}^{3}$ & $133.29 \pm 16.39$ & $137.95 \pm 18.66$ & 0.462 \\
\hline Lat parietal vol., $\mathrm{cm}^{3}$ & $121.92 \pm 12.49$ & $127.31 \pm 16.98$ & 0.337 \\
\hline Med parietal vol., $\mathrm{cm}^{3}$ & $68.48 \pm 7.04$ & $70.41 \pm 8.83$ & 0.513 \\
\hline Occipital vol., $\mathrm{cm}^{3}$ & $141.41 \pm 15.41$ & $142.45 \pm 18.75$ & 0.868 \\
\hline Entorhinal cortex vol., $\mathrm{cm}^{3}$ & $2.01 \pm 0.23$ & $2.16 \pm 0.30$ & 0.134 \\
\hline Hippocampal vol., $\mathrm{cm}^{3}$ & $3.13 \pm 0.32$ & $3.37 \pm 0.32$ & $0.039 *$ \\
\hline Lacune, $n$ & $2.25 \pm 4.16$ & $1.91 \pm 4.69$ & 0.827 \\
\hline Cortical microbleed, $n$ & $2.22 \pm 3.19$ & $0.27 \pm 0.83$ & 0.105 \\
\hline Periventricular WMH, G1/2/3 & $3 / 7 / 2$ & $25 / 6 / 4$ & 0.009 \\
\hline Deep WMH, G1/2/3 & $6 / 5 / 1$ & $28 / 6 / 1$ & 0.126 \\
\hline \multicolumn{4}{|l|}{ Cognition and self-reported symptoms } \\
\hline Cognitive complaints (CFQ total score) & $31.09 \pm 19.92$ & $28.50 \pm 12.51$ & 0.691 \\
\hline Existence of subjective concern about cognitive decline, $n, \%$ & $8 / 11,72.7$ & $30 / 34,88.2$ & 0.337 \\
\hline Existence of informant's report a decline, $n, \%$ & $8 / 11,72.7$ & $15 / 34,44.1$ & 0.099 \\
\hline Symptom's onset, years & $70.27 \pm 7.51$ & $64.29 \pm 7.32$ & 0.024 \\
\hline K-MMSE, total score & $27 \pm 2.05$ & $27.69 \pm 1.81$ & 0.279 \\
\hline Digit span_attention, $\%$ & $55.16 \pm 28.54$ & $67.54 \pm 30.64$ & 0.226 \\
\hline Boston naming test, \% & $66.24 \pm 18.89$ & $57.22 \pm 24.82$ & 0.258 \\
\hline SVLT immediate recall, \% & $37.20 \pm 20.24$ & $48.44 \pm 22.79$ & 0.137 \\
\hline SVLT_delayed recall, \% & $47.82 \pm 20.71$ & $41.57 \pm 23.24$ & 0.414 \\
\hline SVLT_recognition, \% & $51.01 \pm 22.99$ & $45.93 \pm 25.73$ & 0.549 \\
\hline RCFT_copy, \% & $76.01 \pm 11.41$ & $66.98 \pm 20.52$ & 0.156 \\
\hline RCFT_immediate recall, \% & $66.28 \pm 29.81$ & $53.77 \pm 21.31$ & 0.123 \\
\hline RCFT_delayed recall, \% & $66.45 \pm 26.70$ & $50.80 \pm 22.68$ & 0.056 \\
\hline RCFT_recognition, \% & $46.85 \pm 25.62$ & $48.67 \pm 27.92$ & 0.844 \\
\hline COWAT_phonemic, \% & $47.83 \pm 27.09$ & $46.29 \pm 23.94$ & 0.853 \\
\hline Stroop_color reading, \% & $52.38 \pm 33.87$ & $60.15 \pm 27.38$ & 0.429 \\
\hline
\end{tabular}

PET, positron emission tomography; APOE4, apolipoprotein $\varepsilon 4$; DM, diabetes mellitus; SUVR, standardized uptake value ratio; vol, volume measured using QBraVo program; CFQ, cognitive failure questionnaire; WMH, white matter hyperintensities; K-MMSE, Korean version of the mini-mental state examination; SVLT, Seoul Verbal Learning Test; RCFT, Rey Complex Figure Test; COWAT, Controlled Oral Word Association Test. * The statistical significance disappeared after adjustment for age. \%: percentile scores (0-100) after adjustment for age, gender, and education. Higher scores indicate better cognition. 
Table 2. Baseline characteristics and clinical findings between groups (follow-up completers)

\begin{tabular}{|c|c|c|c|}
\hline Variables & $\begin{array}{l}\text { Amyloid PET+ } \\
(n=9)\end{array}$ & $\begin{array}{l}\text { Amyloid PET- } \\
(n=22)\end{array}$ & $p$ value \\
\hline \multicolumn{4}{|l|}{ Demographics } \\
\hline Age, years & $73.78 \pm 4.52$ & $68.27 \pm 6.92$ & 0.037 \\
\hline Symptom's duration, years & $4.69 \pm 5.17$ & $3.64 \pm 4.12$ & 0.554 \\
\hline Female, $n, \%$ & $5,55.6$ & $15,68.2$ & 0.548 \\
\hline Education, years & $7.33 \pm 2.18$ & $9.80 \pm 5.20$ & 0.074 \\
\hline APOE4 allele, $n, \%$ & $4,44.4$ & $4,18.2$ & 0.185 \\
\hline Hypertension, $n, \%$ & $4,44.4$ & $10,45.5$ & 0.637 \\
\hline $\mathrm{DM}, n, \%$ & $3,33.3$ & $6,27.3$ & 0.528 \\
\hline Hyperlipidemia, $n, \%$ & $3,33.3$ & $5,22.7$ & 0.424 \\
\hline \multicolumn{4}{|l|}{ Neuroimaging } \\
\hline Global SUVR, ratio & $1.53 \pm 0.14$ & $1.17 \pm 0.05$ & $<0.001$ \\
\hline Hippocampal atrophy, grade & $0.55 \pm 0.53$ & $1.00 \pm 0.87$ & 0.084 \\
\hline Ant frontal vol., $\mathrm{cm}^{3}$ & $50.26 \pm 6.94$ & $55.91 \pm 7.20$ & 0.077 \\
\hline Dorsolateral frontal vol., $\mathrm{cm}^{3}$ & $66.25 \pm 8.04$ & $71.06 \pm 9.51$ & 0.237 \\
\hline Orbitofrontal vol., $\mathrm{cm}^{3}$ & $33.13 \pm 4.82$ & $36.85 \pm 5.33$ & 0.110 \\
\hline Ant temporal vol., $\mathrm{cm}^{3}$ & $29.85 \pm 4.25$ & $32.44 \pm 5.79$ & 0.284 \\
\hline Med temporal vol., $\mathrm{cm}^{3}$ & $30.91 \pm 3.48$ & $32.50 \pm 3.46$ & 0.297 \\
\hline Lat temporal vol., $\mathrm{cm}^{3}$ & $132.50 \pm 14.76$ & $137.64 \pm 17.68$ & 0.492 \\
\hline Lat parietal vol., $\mathrm{cm}^{3}$ & $118.79 \pm 11.00$ & $126.21 \pm 14.87$ & 0.234 \\
\hline Med parietal vol., $\mathrm{cm}^{3}$ & $65.82 \pm 5.48$ & $70.23 \pm 9.08$ & 0.236 \\
\hline Occipital vol., $\mathrm{cm}^{3}$ & $141.41 \pm 16.17$ & $143.07 \pm 19.08$ & 0.836 \\
\hline Entorhinal cortex vol., $\mathrm{cm}^{3}$ & $1.95 \pm 0.24$ & $2.20 \pm 0.29$ & 0.050 \\
\hline Hippocampal vol., $\mathrm{cm}^{3}$ & $3.16 \pm 0.40$ & $3.34 \pm 0.25$ & 0.155 \\
\hline Lacune, $n$ & $1.22 \pm 1.20$ & $2.86 \pm 5.73$ & 0.214 \\
\hline Cortical microbleed, $n$ & $1.71 \pm 2.63$ & $0.16 \pm 0.69$ & 0.170 \\
\hline Periventricular WMH, G1/2/3 & $3 / 4 / 2$ & $14 / 5 / 3$ & 0.326 \\
\hline Deep WMH, G1/2/3 & $5 / 4 / 0$ & $17 / 4 / 1$ & 0.325 \\
\hline \multicolumn{4}{|l|}{ Cognition and self-reported symptoms } \\
\hline Cognitive complaints (CFQ total score) & $28.00 \pm 18.46$ & $28.95 \pm 13.12$ & 0.876 \\
\hline Existence of subjective concern about cognitive decline, $n(\%)$ & $5(71.43)$ & $20(86.96)$ & 0.699 \\
\hline Existence of informant's report a decline, $n(\%)$ & $6(85.71)$ & $13(56.52)$ & 0.339 \\
\hline Symptom's onset, yr & $70.14 \pm 7.47$ & $64.57 \pm 7.58$ & 0.099 \\
\hline K-MMSE, total score & $27.22 \pm 1.79$ & $27.50 \pm 1.90$ & 0.710 \\
\hline Digit span_attention, \% & $55.64 \pm 30.41$ & $68.10 \pm 31.36$ & 0.320 \\
\hline Boston naming test, \% & $66.97 \pm 17.25$ & $62.11 \pm 23.12$ & 0.575 \\
\hline SVLT immediate recall, \% & $31.33 \pm 14.43$ & $49.50 \pm 21.13$ & 0.026 \\
\hline SVLT_delayed recall, \% & $49.37 \pm 22.38$ & $43.19 \pm 23.07$ & 0.501 \\
\hline SVLT_recognition, \% & $47.45 \pm 17.40$ & $50.38 \pm 22.10$ & 0.726 \\
\hline RCFT_copy, \% & $73.58 \pm 11.95$ & $67.71 \pm 20.66$ & 0.434 \\
\hline RCFT_immediate recall, \% & $61.83 \pm 32.54$ & $55.55 \pm 23.68$ & 0.556 \\
\hline RCFT_delayed recall, \% & $62.92 \pm 28.01$ & $52.03 \pm 25.24$ & 0.303 \\
\hline RCFT_recognition, \% & $48.78 \pm 29.18$ & $54.49 \pm 26.65$ & 0.605 \\
\hline COWAT_phonemic, \% & $44.35 \pm 21.62$ & $46.65 \pm 24.17$ & 0.806 \\
\hline Stroop_color reading, \% & $53.82 \pm 35.07$ & $59.72 \pm 26.81$ & 0.615 \\
\hline
\end{tabular}

\%: percentile scores (0-100) after adjustment for age, gender, and education. Higher scores indicate better cognition. PET, positron emission tomography; APOE4, apolipoprotein ع4; DM, diabetes mellitus; SUVR, standardized uptake value ratio; vol, volume measured using QBraVo program; CFQ, cognitive failure questionnaire; WMH, white matter hyperintensities; K-MMSE, Korean version of the mini-mental state examination; SVLT, Seoul Verbal Learning Test; RCFT, Rey Complex Figure Test; COWAT, Controlled Oral Word Association Test.

Faster Memory Decline in AmyloidPositive Subjective Cognitive Decline
Dement Geriatr Cogn Disord 2021;50:437-445 DOI: $10.1159 / 000519766$ 
Table 3. Cognitive and neurodegenerative changes in each group (subjects who completed follow-up)

\begin{tabular}{llllll}
\hline Variables & $\mathrm{A}+\mathrm{SCD}(n=9)$ & $\mathrm{A}-\mathrm{SCD}(n=22)$ & $p$ value & $\begin{array}{l}\text { Model 1 } \\
p \text { value }\end{array}$ & $\begin{array}{l}\text { Model } 2 \\
p \text { value }\end{array}$ \\
\hline K-MMSE score change & $-1.13 \pm 2.85$ & $-0.55 \pm 2.82$ & 0.624 & 0.588 & 0.771 \\
SVLT-delayed recall \% change & $-4.69 \pm 33.03$ & $18.11 \pm 21.75$ & 0.036 & 0.018 & 0.075 \\
Entorhinal vol. change, $\mathrm{cm}^{3}$ & $0.16 \pm 0.28$ & $-0.06 \pm 0.20$ & 0.036 & 0.031 & 0.117 \\
Hippocampal vol. change, $\mathrm{cm}^{3}$ & $0.07 \pm 0.08$ & $0.06 \pm 0.14$ & 0.787 & 0.390 & 0.402 \\
\hline
\end{tabular}

Model 1: adjusted for age. Model 2: adjusted for age, educational level, and baseline scores (K-MMSE, and SVLTdelayed recall scores). $A+S C D$, amyloid-positive subjective cognitive decline; $A-S C D$, amyloid-negative subjective cognitive decline; PET, positron emission tomography; K-MMSE, Korean version of mini-mental state examination; SVLT, Seoul Verbal Learning Test.

participants did not undergo follow-up evaluations due to refusal $(n=14)$ or moving to other regions $(n=2)$. At baseline, 35 participants $(74.4 \%)$ were negative and the other $12(25.6 \%)$ were positive for amyloid deposition based on visual ratings of florbetaben PET scans. Baseline demographics and clinical characteristics $(n=47)$ are shown in Table 1 . The A+SCD participants were older $(p=0.001)$, had more advanced hippocampal atrophy $(p=0.015)$, and showed more periventricular WMH ( $p=$ 0.009) compared with A-SCD participants (Table 1). Baseline regional volumes (adjusted for age), small vessel disease findings, and neuropsychological test results (adjusted for age, sex, and educational level) were similar between the 2 groups (Table 1).

Baseline characteristics of the participants who completed follow-up evaluations $(n=31)$ are shown in Table 2. Among participants who completed the study, A + SCD participants $(n=9)$ were older and had lower verbal immediate recall scores (Table 2); 1 participant was excluded from quantitative PET imaging analysis due to poor imaging quality. Mean follow-up duration was 24.7 \pm 7.5 months (range, 10-36 months). Participants who completed the study $(n=31)$ did not differ from subjects who dropped out $(n=16)$ in terms of baseline clinical characteristics such as age, sex, educational level, comorbidities, and cognitive scores (online suppl. Table 1).

\section{Cognitive Decline and Medial Temporal}

Neurodegenerations

During the study period, no participant progressed to MCI or dementia. K-MMSE scores decreased by a mean of 1.13 points in the $A+S C D$ group and 0.55 points in the A-SCD group, although the differences did not reach statistical significance ( $p>0.05$, Table 3$)$. However, after adjustment for age, A+SCD participants showed greater de- clines of verbal memory delayed recall function (SVLTdelayed recall score) than the A-SCD participants (Table 3). After adjustment for age, educational level, and baseline cognitive scores, SVLT-delayed recall scores showed a trend of more rapid decline in A+SCD participants compared with A-SCD participants (Table 3). Entorhinal cortical volume, a biomarker representing neurodegenerations, decreased more in A-SCD participants after adjustment for age although the volume changes were small (Table 3). Other variables including GDS, CDR, CDR sum of boxes score, and K-IADL scores did not show significant differences between the 2 groups during the study period (data not shown).

\section{Correlations between Baseline Factors and Cognitive Decline}

It was assessed whether baseline amyloid burden was the most relevant factor associated with future changes in SVLT-delayed recall score compared with other clinical factors and imaging biomarkers. In univariable regression analysis, baseline SVLT-delayed recall score, baseline number of microbleeds, and global SUVR values were the most relevant factors associated with changes in SVLT-delayed recall score (Table 4). Other baseline characteristics such as demographics, other cognitive scores, severity of the cognitive complaints, and neuroimaging biomarkers such as hippocampal volumes or small vessel disease markers were not related with SVLT-delayed recall score changes (Table 4). Because the baseline number of microbleeds was significantly associated with global SUVR values ( $p=0.004, r=0.541)$, the number of microbleeds was not included in the multivariable analysis. Global SUVR values and baseline SVLT-delayed recall scores were not correlated with each other $(p>0.05)$. In multivariable regression analysis, baseline SVLT score 
Table 4. Correlations between baseline factors and verbal memory decline during 2 years

\begin{tabular}{|c|c|c|c|c|c|c|}
\hline \multirow[t]{2}{*}{ Variables } & \multicolumn{3}{|c|}{ Univariable } & \multicolumn{3}{|c|}{ Multivariable } \\
\hline & $\beta$ & $95 \% \mathrm{Cl}$ & $p$ value & $\beta$ & $95 \% \mathrm{Cl}$ & $p$ value \\
\hline Global SUVR (ratio) & -0.488 & $-125.121 \sim-22.675$ & 0.006 & -0.354 & $-102.080 \sim-5.352$ & 0.031 \\
\hline Microbleed $(n)$ & -0.429 & $-13.934 \sim 0.829$ & 0.029 & & & \\
\hline Age (years) & 0.056 & $-1.265 \sim 1.707$ & 0.764 & & & \\
\hline Female gender & -0.189 & $-30.525 \sim 9.961$ & 0.307 & & & \\
\hline Education (years) & 0.134 & $-1.377 \sim 2.908$ & 0.471 & & & \\
\hline Baseline SVLT delayed recall (\%) & -0.543 & $-1.033 \sim-0.259$ & 0.002 & -0.435 & $-0.8997 \sim-0.137$ & 0.009 \\
\hline Baseline CFQ score & -0.254 & $-1.134 \sim 0.207$ & 0.168 & & & \\
\hline Concern about cognitive decline & 0.009 & $-26.610 \sim 27.855$ & 0.963 & & & \\
\hline Informant's report a decline & -0.280 & $-35.475 \sim 4.957$ & 0.133 & & & \\
\hline APOE4 & 0.098 & $-16.614 \sim 28.256$ & 0.600 & & & \\
\hline Lacune $(n)$ & 0.003 & $-2.030 \sim 2.065$ & 0.986 & & & \\
\hline WMH, periventricular & -0.222 & $-20.552 \sim 5.159$ & 0.231 & & & \\
\hline WMH, deep & 0.032 & $-16.957 \sim 20.107$ & 0.863 & & & \\
\hline EC vol. $\left(\mathrm{cm}^{3}\right)$ & 0.037 & $-32.012 \sim 38.736$ & 0.847 & & & \\
\hline Hippocampal vol. $\left(\mathrm{cm}^{3}\right)$ & 0.058 & $-29.869 \sim 40.314$ & 0.763 & & & \\
\hline
\end{tabular}

$\%$ : percentile scores (0 100) after adjustment for age, gender, and education. Higher scores indicate better cognition. CFQ, cognitive failure questionnaire; APOE4, apolipoprotein $\varepsilon 4$; WMH, white matter hyperintensities; MMSE, mini-mental state examination; SUVR, standardized uptake value ratio; SVLT, Seoul verbal learning test; EC, entorhinal cortex; vol, volume measured using the QBraVo program.

and global SUVR value were the baseline factors associated with change in SVLT memory score (see Table 4). Baseline global SUVR value had an inverse relationship with verbal memory decline (Table 4).

\section{Discussion}

This prospective observational cohort study was conducted in participants with SCD during a 24 -month period and tested whether cognitive trajectories in $\mathrm{A}+\mathrm{SCD}$ participants were different from those in A-SCD participants. In addition, the baseline factors associated with memory decline were assessed. Major findings of the current study showed that $\mathrm{A}+\mathrm{SCD}$ participants experienced more rapid decline in verbal memory function compared with A-SCD participants. Furthermore, baseline amyloid burden was associated with verbal memory decline during the relatively short study period.

Participants in the 2 groups did not progress to MCI or dementia during the study period. Consistent with the clinical outcomes, ADL and CDR scores did not change at the study endpoint, as was expected, considering that the study participants were cognitively normal at baseline, and that clinical progression rates from SCD to MCI/ dementia are only $5-10 \%$ annually $[4,11,22]$. However, general cognitive scores based on K-MMSE differed between $\mathrm{A}+\mathrm{SCD}$ and $\mathrm{A}-\mathrm{SCD}$ participants. Notably, verbal memory scores based on SVLT-delayed recall tests showed significantly different changes between the 2 groups; greater verbal memory decline was observed in SCD participants with Alzheimer's pathologic changes despite the relatively short follow-up duration and agreement in baseline cognitive scores. Verbal memory delayed recall function might be associated with medial temporal function and decrease early and prominently in typical AD. Consistent with greater memory score changes in $A+S C D$ participants, amyloid deposition, rather than other baseline factors, was a relevant risk factor associated with verbal memory decline. Furthermore, amyloid burden was associated more highly with memory changes than were other baseline factors such as entorhinal/hippocampal atrophy, small vessel disease markers (lacunes/WMHs/microbleeds), or age, all of which are known to be associated with verbal memory function. Based on previous evidence, neurodegenerations are known to be closely linked to cognitive decline while amyloid burden is not strongly related with short-term cognitive decline [23]. During the study period, entorhinal volume showed more decrease in A-SCD participants 
compared with that in $\mathrm{A}+\mathrm{SCD}$ participants, although the volume differences were numerically small and the entorhinal/hippocampal volume changes during the study period were not definite. The conflicting result can be explained that neurodegenerative changes are not definite out considering amyloidosis may not cause aggressive progressions; neurodegenerations only combined with amyloid pathologies would likely show cognitive progresloid burden, rather than neurodegenerative changes, ory decline in this very early stage of $\mathrm{AD}$. In summary, assessment for existence of cognitive complaints and brain amyloidosis may enable predicting faster memory decline even in a short-term period.

The present study had a few limitations. First, followup duration was relatively short considering that annual progression rate of SCD is below $10 \%$ based on previous large cohort studies. Second, other risk factors such as tau-related biomarkers, lifestyle factors associated with clinical progression, and combined non-AD related pathologies such as Parkinson's disease were not studied. Because SCD is a heterogeneous condition with multiple pathologies, mood disorders, and personality factors, future studies should adopt detailed pathologic biomarkers and longer follow-up duration. The last, sampling bias might exist because this study was conducted in a single center, hence, generalization of the results need cautious interpretations.

Despite the few study limitations, the results have strength because participants were consecutively recruited using comprehensive neuropsychological test battery and underwent multiple biomarker evaluations. All participants underwent amyloid PET scans and regular brain MRI follow-ups and quantitative measures for amyloid burden and neurodegenerative changes. In addition, we assessed the intensity of subjective cognitive complaints and characteristics of the cognitive complaints using SCD-plus criteria although they did not show significant effects on cognitive declines. There have been few studies that investigated longitudinal cognitive trajectories of SCD based on baseline amyloid burden, neurodegenerations, and detailed clinical evaluations. The present results imply that faster verbal memory decline can be predicted in cognitively unimpaired elderly with both brain amyloidosis and SCD, which should be confirmed in future studies with long-term follow-ups. yet in this early stage and neurodegeneration alone withsion in elderly participants with SCD [24]. Hence, amycould be the more important factor affecting future mem-

\section{Statement of Ethics}

This study was conducted in accordance with the Declaration of Helsinki and the guidelines on good clinical practice. All eligible patients who had signed the consent form were included in the study. The study protocol was validated by the Dong-A University Hospital's Ethics Committee (DAUHIRB-16-232), Busan, South Korea.

\section{Conflict of Interest Statement}

The authors declare that they have no competing interests.

\section{Funding Sources}

This study was supported by Biomedical Research Institute Grant (2018B031) of Pusan National University Hospital, the National Research Foundation of Korea (NRF) grant funded by the Korea government (MSIT) (2020R1C1C1006121), and the Ministry of Health and Welfare, HI18C0530.

\section{Author Contributions}

Y.J.H. and K.W.P. contributed to the study concept and design. Y.J.H. analyzed and interpreted the results. Y.J.H. drafted the manuscript. J.W.P., S.B.L., S.-H.K., Y.K., D.-W.R., K.W.P., and D.W.Y. were involved in data collection, recruitment, and evaluation of the patients. All authors read and approved the final manuscript.

\section{Data Availability Statement}

All data generated or analyzed during this study are included in this article and its online supplementary material file. Further inquiries can be directed to the corresponding author on reasonable request.
References
1 Rönnlund M, Sundström A, Adolfsson R, Nilsson LG. Subjective memory impairment in older adults predicts future dementia independent of baseline memory performance: evidence from the Betula Prospective Cohort Study. Alzheimers Dement. 2015;11:1385-92.

2 Kielb S, Rogalski E, Weintraub S, Rademaker A. Objective features of subjective cognitive decline in a United States national database. Alzheimers Dement. 2017;13:1337-44.

3 Hong YJ, Lee JH. Subjective cognitive decline and alzheimer's disease spectrum disorder. Dement Neurocogn Disord. 2017;16(2):40-7.

4 Slot RER, Sikkes SAM, Berkhof J, Brodaty H, Buckley R, Cavedo E, et al. Subjective cognitive decline and rates of incident alzheimer's disease and non-alzheimer's disease dementia. Alzheimers Dement. 2019;15:465-76. 
5 Jessen F, Amariglio RE, Buckley RF, van der Flier WM, Han Y, Molinuevo JL, et al. The characterisation of subjective cognitive decline. Lancet Neurol. 2020;19:271-8.

6 van Harten AC, Visser PJ, Pijnenburg YA, Teunissen CE, Blankenstein MA, Scheltens P, et al. Cerebrospinal fluid $A \beta 42$ is the best predictor of clinical progression in patients with subjective complaints. Alzheimers Dement. 2013;9:481-7.

7 Hong YJ, Yoon B, Shim YS, Kim SO, Kim HJ, Choi SH, et al. Predictors associated with the clinical progression of elderly with subjective memory impairment: data from the clinical research centers for dementia of South Korea (CREDOS). Dement Geriatr Cogn Disord. 2015;40(3-4):158-65.

8 Risacher SL, Kim S, Nho K, Foroud T, Shen L, Petersen RC, et al. APOE effect on Alzheimer's disease biomarkers in older adults with significant memory concern. Alzheimers Dement. 2015;11:1417-29.

9 Hong YJ, Park KW, Kang DY, Lee JH. Prediction of alzheimer's pathological changes in subjective cognitive decline using the self-report questionnaire and neuroimaging biomarkers. Dement Neurocogn Disord. 2019; 18:19-29.

10 Jessen F, Amariglio RE, van Boxtel M, Breteler M, Ceccaldi M, Chételat G, et al. A conceptual framework for research on subjective cognitive decline in preclinical Alzheimer's disease. Alzheimers Dement. 2014;10:84452.

11 Kang Y, Jahng S, Na DL. Seoul neuropsychological screening battery. 2nd edn. Seoul: $\mathrm{Hu}$ - man Brain Research \& Consulting Co; 2012.

12 Morris JC. The clinical dementia rating (CDR): current version and scoring rules. Neurology. 1993;43:2412-4.

13 Noh Y, Lee Y, Seo SW, Jeong JH, Choi SH, Back JH, et al. A new classification system for ischemia using a combination of deep and periventricular white matter hyperintensities. J Stroke Cerebrovasc Dis. 2014;23:63642.

14 Wardlaw JM, Smith EE, Biessels GJ, Cordonnier C, Fazekas F, Frayne R, et al. Neuroimaging standards for research into small vessel disease and its contribution to ageing and neurodegeneration. Lancet Neurol. 2013;12: $822-38$.

15 Scheltens P, Leys D, Barkhof F, Huglo D, Weinstein HC, Vermersch P, et al. Atrophy of medial temporal lobes on MRI in 'probable' alzheimer's disease and normal ageing: diagnostic value and neuropsychological correlates. J Neurol Neurosurg Psychiatry. 1992; 55(10):967-72.

16 Becker GA, Ichise M, Barthel H, Luthardt J, Patt $M$, Seese A, et al. PET quantification of $18 \mathrm{~F}$-florbetaben binding to $\beta$-amyloid deposits in human brains. J Nucl Med. 2013;54: 723-31.

17 Barthel H, Gertz HJ, Dresel S, Peters O, Bartenstein P, Buerger K, et al. Cerebral amyloid-beta PET with florbetaben (18F) in patients with alzheimer's disease and healthy controls: a Multicentre Phase 2 Diagnostic Study. Lancet Neurol. 2011;10(5):424-35.

18 Ye BS, Seo SW, Kim GH, Noh Y, Cho H, Yoon $\mathrm{CW}$, et al. Amyloid burden, cerebrovascular disease, brain atrophy, and cognition in cognitively impaired patients. Alzheimers Dement. 2015;11:494-e3.

19 Han C, Jo SA, Jo I, Kim E, Park MH, Kang Y. An adaptation of the Korean mini-mental state examination (K-MMSE) in elderly Koreans: demographic influence and populationbased norms (the AGE Study). Arch Gerontol Geriatr. 2008;47:302-10.

20 Broadbent DE, Cooper PF, FitzGerald P, Parkes KR. The cognitive failures questionnaire (CFQ) and its correlates. Br J Clin Psychol. 1982;21:1-16.

21 Won CW, Rho YG, Sunwoo D, Lee YS. The validity and reliability of Korean instrumental activities of daily living (K-IADL) scale. J Korean Geriatr Soc. 2002;6(4):273-80.

22 Snitz BE, Wang T, Cloonan YK, Jacobsen E, Chang $\mathrm{CH}$, Hughes TF, et al. Risk of progression from subjective cognitive decline to mild cognitive impairment: the Role of Study Setting. Alzheimers Dement. 2018;14:734-42.

23 Dubois B, Epelbaum S, Nyasse F, Bakardjian H, Gagliardi G, Uspenskaya O, et al. Cognitive and neuroimaging features and brain $\beta$-amyloidosis in individuals at risk of alzheimer's disease (INSIGHT-preAD): a Longitudinal Observational Study. Lancet Neurol. 2018;17(4):335-46.

24 Zhao Y, Tudorascu DL, Lopez OL, Cohen AD, Mathis CA, Aizenstein HJ, et al. Amyloid $\beta$ deposition and suspected non-alzheimer pathophysiology and cognitive decline patterns for 12 Years in oldest old participants without dementia. JAMA Neurol. 2018;75: 88-96. 